\title{
On the Mode of the Conversion of Elymoclavine to Agroclavine*
}

Sir :

Previously, we ${ }^{1)}$ found that agroclavine (IV) exclusively yielded a basic substance, lysergine, on treatment with hot sodium butylate.

Recently, we have treated elymoclavine (I) with sodium butylate by a similar procedure as in the case of agroclavine in the hope that a certain derivative corresponding to lysergine would result.

This treatment gave a brown colored mixture from which two basic substances $A$ and $B$ were isolated. A was obtained in a very poor yield; colorless prisms from ethanol, m.p. $249-250^{\circ}$ (uncorr. decomp.), $\left.[\alpha]\right]_{\mathrm{D}}^{37}=+50^{\circ}$, $[\alpha]_{6461}^{27}=+85^{\circ}$ (c, 0.3 in pyridine). Anal. Found: C, 75.68; H, 7.21; N, 10.92. Calcd. for $\mathrm{C}_{16} \mathrm{H}_{18} \mathrm{ON}_{3}: \quad \mathrm{C}, 75.55 ; \mathrm{H}, 7.14 ; \mathrm{N}, 11.02 \%$. This substance was just identical with lysergol (II) not only in crystal form, melting point and specific rotation, but also in color reaction, paper chromatographic behavior and UV.and IR.-spectra. B was obtained in an excellent yield; colorless needles or prisms from ethyl acetate, acetone, methanol or ethanol, m.p. $244-245^{\circ}$ (uncorr. decomp.), $[\alpha]_{\mathrm{D}}^{00}=+407^{\circ}$, $[\alpha]_{5461}^{* 0}=+571^{\circ}$ (c, 0.18 in $\left.\mathrm{CHCl}_{3}\right), \quad[\alpha]_{\mathrm{D}}^{20}=$ $+463^{\circ},[\alpha]_{5461}^{20}=+630^{\circ}$ (c, 0.23 in pyridine). Anal. Found: C, $81.46 ; \mathrm{H}, 7.00 ; \mathrm{N}, 11.69 ; \mathrm{N}-$ $\mathrm{CH}_{3}$, 11.94. Calcd. for $\mathrm{C}_{16} \mathrm{H}_{16} \mathrm{~N}_{2}$ : C, 81.32;
$\mathrm{H}, 6.82 ; \mathrm{N}, 11.82 ; \mathrm{N}-\mathrm{CH}_{3}, 12.29 \%$. It gave a yellowish-green color, undistinguishable from that given by molliclavine? with Allport and Cocking's reagent, but, unlike the latter, it gave almost no color with concd. sulfuric acid. The UV.-spectrum of this substance was considerably peculiar, showing maxima at the vicinity of 243,263 and $335 \mathrm{~m} \mu$. It was insoluble in petroleum ether, sparingly soluble in benzene, ether, ethyl acetate, acetone, methanol and ethanol, but moderately soluble in chloroform and pyridine. Its solutions in organic solvents exhibited a marked fluorescence. It was almost insoluble in water, but readily soluble in dilute acids. As in both cases of agroclavine and lysergine, this substance exclusively yielded festuclavine (alkaloid " $\mathrm{Y}$ ") on catalytic hydrogenation, whereas, it yielded the same substance together with agroclavine, lysergine, pyroclavine ${ }^{3)}$ and costaclavine ${ }^{3)}$ on reduction with sodium and $n$-butanol. Moreover, this substance itself was obtained in an excellent yield by the treatment of lysergol (II) with hot sodium butylate. These data indicate that this substance possesses the formula III. We have therefore adopted the designation lysergene for this basic product.

Formerly, we ${ }^{1)}$ found that elymoclavine (I) was converted into agroclavine (IV) by reduc-

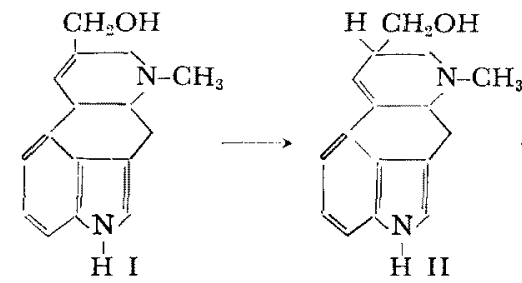

* This work was reported at the Meeting of Agr. Chem. Soc. Japan held at Mie University on October 21, 1956.

1) S. Yamatodani and M. Abe, This Bulletin (communication), 20, $95(1956)$.

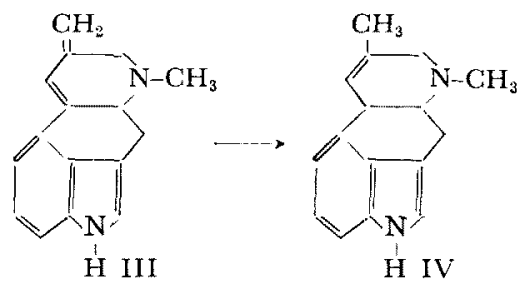

2) M. Abe and S. Yamatodani, This Bulletin (communication), 19, 161 (1955).

3) M. Abe, S. Yamatodani, T. Yamano and M. Kusumoto, This Bulletin (communication), 20, 59 (1956). 
tion with sodium and $n$-butanol. From the results described above, it is evident that the conversion was performed through the process shown here.

Up to the present, lysergol (II) as well as lysergene (III) has not been found either in the sclerotium or in the saprophytic culture of ergot fungus. It is, however, presumed that agroclavine must be produced also in the living cells through the same process as shown above.

Saburō Yamatodani Matazō ABE

The Laboratory of the Institute for Fermentation, attached to Takeda Pharmaceutical Industries, Ltd. Osaka, Japan

Received March 20, 1957 